\title{
Point-to-point connectivity, an abstract concept or a key issue for risk assessment studies?
}

\author{
Paolo Trinchero*, Xavier Sánchez-Vila, Daniel Fernàndez-Garcia \\ Department of Geotechnical Engineering and Geosciences, Technical University of Catalonia, C/Jordi Girona 1-3, 08034 Barcelona, Spain
}

\section{A R T I C L E I N F O}

\section{Article history:}

Received 11 March 2008

Received in revised form 29 July 2008

Accepted 2 September 2008

Available online 11 September 2008

\section{Keywords:}

Point-to-point connectivity

Connectivity patterns

Capture zones

Heterogeneity

\begin{abstract}
A B S T R A C T
Connectivity of high/low-permeability areas has been recognized to significantly impact groundwater flow and solute transport. The task of defining a rigorous quantitative measure of connectivity for continuous variables has failed so far, and thus there exist a suite of connectivity indicators which are dependent on the specific hydrodynamic processes and the interpretation method. Amongst the many existing indicators, we concentrate on those characterizing connectivity between the points involved in a hydraulic or tracer test. The flow connectivity indicator used here is based on the time elapsed for hydraulic response in a pumping test (e.g., the storage coefficient estimated by the Cooper-Jacob method, $S_{\text {est }}$ ). Regarding transport, we select the estimated porosity from the breakthrough curve $\left(\phi_{\text {est }}\right)$. According to Knudby and Carrera [Knudby C, Carrera J. On the relationship between indicators of geostatistical, flow and transport connectivity. Adv Water Resour 2005;28(4):405-21] these two indicators measure connectivity differently, and are poorly correlated. Here, we use perturbation theory to analytically investigate the intrinsic relationship between $S_{\text {est }}$ and $\phi_{\text {est }}$. We find that $\phi_{\text {est }}$ can be expressed as a weighted line integral along the particle trajectory involving two parameters: the transmissivity point values, $T$, and the estimated values of $S_{\text {est }}$ along the particle path. The weighting function is linear with the distance from the pumping well, thus the influence of the weighting function is maximum at the injection area, whereas the hydraulic information close to the pumping well becomes redundant (null weight). The relative importance of these two factors is explored using numerical simulations in a given synthetic aquifer and tested against intermediate-scale laboratory tracer experiments. We conclude that the degree of connectivity between two points of an aquifer (point-to-point connectivity) is a key issue for risk assessment studies aimed at predicting the travel time of a potential contaminant.
\end{abstract}

(c) 2008 Elsevier Ltd. All rights reserved.

\section{Introduction}

Although in the last 20 years many efforts have been devoted to define and assess the impact of hydraulic connectivity in groundwater flow and solute transport, limited visible results are appreciable in raising the policy makers and the professionals awareness of its importance in risk assessment studies. The main reasons can be associated with the lack of a unique definition of connectivity and the difficulty of properly characterize the geological features of the medium.

The first evidence of the role of hydraulic connectivity in flow and transport processes arose in the 1980s with the blossoming of groundwater studies in fractured media. A key idea was presented by de Marsily [10] who argued that only a small subset of

\footnotetext{
* Corresponding author. Tel.: +34 9340172 44; fax: +34 934017251.

E-mail addresses: paolo.trinchero@upc.edu, paolo.trinchero@gmail.com (P. Trinchero),xavier.sanchez-vila@upc.edu (X. Sánchez-Vila), daniel.fernandez.g@ upc.edu (D. Fernàndez-Garcia).
}

the fractures was actually contributing to total flow and transport. The idea was soon transferred to porous media. Fogg [14] carried out a numerical study of the three-dimensional distribution of hydraulic conductivity, $K$, in the Wilcox aquifer system. He argued that the flow system in the aquifer was mainly controlled by the continuity and interconnectedness of the sand, rather than by their actual local hydraulic conductivity values. Poeter and Townsend [29] studied a two-dimensional section of a fluvial aquifer having highly connected hydraulic features. They found that the travel time estimates exclusively calculated based on the effective conductivity associated with extreme values were more consistent with field observations. In a later work, LaBolle and Fogg [23] showed that connectivity of low-permeability hydrofacies is an important process controlling the migration of contaminant plumes and the success of remediation actions.

Sanchez-Vila et al. [33] analyzed the departure from the assumption of multiGaussianity in mapping the spatial variations of aquifer properties through geostatistical models. They found that the presence of structures favoring connectivity of the large local $T$ values yields effective transmissivity values, $T_{\text {eff }}$, larger than 
the geometric mean, $T_{\mathrm{G}}$, the latter value being the effective transmissivity in multiGaussian 2-D fields.

The impact of connectivity of extreme transmissivity values on solute transport was investigated by Gomez-Hernandez and Wen [16], who found that the assumption of multiGaussianity could greatly underestimate travel times. This assumption was further explored by Zinn and Harvey [47]. Well connected fields were found to enhance Darcy-scale mass transfer processes in the system producing tailing in the breakthrough curves, while disconnected fields displayed macrodispersivity values smaller than those observed for multiGaussian fields. Moreover their results regarding effective conductivity reproduce those of Sanchez-Vila et al. [33]. Guimera and Carrera [18] assessed data from 90 tracer test experiments carried out in low-permeability fractured media. They found the effective hydraulic conductivity, estimated as the ratio of water flux to head gradient, to be highly correlated with the apparent porosity (ratio of water velocity to water flux) based on the first arrival time. The authors concluded that first arrival is controlled by the same flow paths as hydraulic conductivity. Zheng and Gorelick [46] studied the effect of having a synthetic fracture network of narrow channels with high conductivity embedded in a homogeneous porous medium. When the contrast of permeability between the channel and the matrix was larger than 100, the spatial concentration of the plume exhibited high asymmetrical non-Gaussian features. On the contrary, when the contrast of permeability was reduced to 30:1, the solute plume approached a Gaussian behavior. High connected flow paths have been shown numerically to lead to

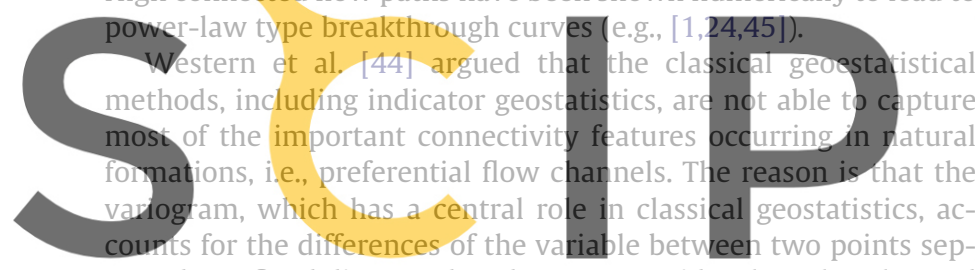
arated at a fixed distance, but does not consider the values located between these two points. To overcome this problem, the authors

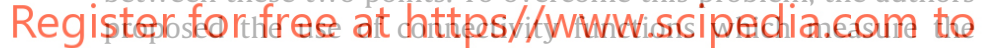
probability that two separate points are connected by a continuous path. Alternatively, the limitation of two-point correlation methods to adequately characterize multimodal distributions have been recently overcome by the development of multiple-point (mp) geostatistics [38]. This technique has been extensively used for the proper characterization of petroleum reservoirs [39,5] as well as for modeling complex aquifer systems [13]. Still, their statistical approach is based on some visual inspection of the geological features and does not include the driven physical processes of flow and transport.

In the same context, Vogel [43] developed a new method to obtain effective hydraulic parameters accounting for the existence of connected paths. This upscaling technique, based on topological indicators, was later applied to unsaturated flow [27]. Recently Kerrou et al. [20] assessed the performance of stochastic multiGaussian techniques to preserve connectivity patterns when characterizing a non-multiGaussian braided channel aquifer. In agreement with Western et al. [44], their results emphasized the need for an adequate description of connectivity, which is not captured by two-point statistics.

A different, albeit related concept, is connectivity of systems based on percolation theory $[2,3,28,25,9]$. In these works, connectivity has a clear physical meaning indicating whether a given fluid is capable of flowing from one point (surface) to another. This process is characterized by the potential existence of a connecting path (classical percolation theory) potentially coupled with the availability of the fluid to invade that particular path (invasion percolation theory). No connection would in a sense mean that there are areas with null transmissivity, and connectivity becomes a geo- metrical feature. Our work focuses on a different approach, since we consider a continuous porous medium, where connectivity is a relative process ("better" or "worse") indicating whether it is possible to connect two points through a preferential flow and/or solute path.

As such, it is obvious that a single quantitative definition of connectivity in heterogeneous porous aquifers is not possible, and so a number of indicators of connectivity has been developed. In a recent study, Knudby and Carrera [21] numerically tested several intuitive indicators of flow and transport connectivity aiming at discerning between well connected and disconnected fields as a whole. They found a weak correlation between flow and transport indicators. Later on, the same authors [22] analyzed the use of apparent diffusivity, $D_{\mathrm{r}}$ (estimated transmissivity divided by estimated storage coefficient), as a measure of connectivity using a Monte Carlo analysis. They found that $D_{\mathrm{r}}$ has a certain degree of correlation with indicators of both transport and flow global connectivity.

As evidenced by this brief review, in the last decades much effort has been dedicated to the study of connectivity as a global property of a field or as an intrinsic feature of fractured systems. Few studies focus on the impact of connectivity patterns on pumping or tracer tests in which the velocity of the signal (heads or concentrations) response between two distinct points is crucial. Schad and Teutsch [36] inferred information on the effective length scale of the heterogeneity structure using pumping test data. Meier et al. [26] analyzed numerically the meaning of the parameters obtained

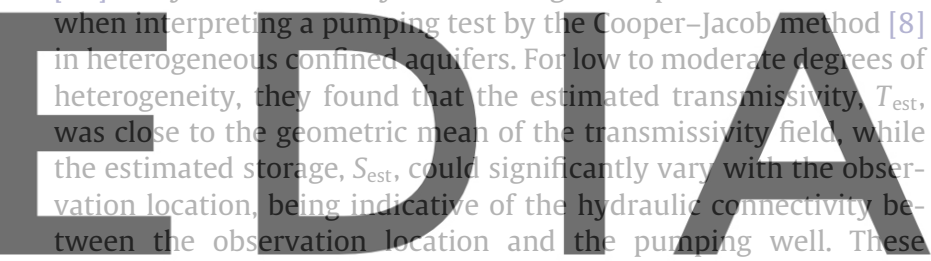
results were confirmed analytically by Sanchez-Vila et al. [34] who obtained an approximate analytical expression for $S_{\text {est }}$ by

Fernandez-Garcia et al. [11] analyzed a suite of convergent-flow tracer tests performed in a reconstructed anisotropic heterogeneous medium. They found that the arrival time (or the estimated porosity) of tracer breakthrough curves was primarily controlled by the preferential flow paths occurring between the pumping well and the injection location, providing little information on the global properties of the transmissivity field, i.e., $T_{\text {eff. Thus, their results }}$ gave an explanation for the observed discrepancy between porosity estimates obtained from field tracer tests and their representative values. This explanation had already been hinted by Sanchez-Vila and Carrera [31] in their analysis of a tracer test performed in a highly heterogeneous strongly anisotropic medium.

An issue to be considered when addressing point-to-point connectivity is the local effect of drilling at the pumping well location. If the well is located in a distinct (high or low) $T$ area, including the possibility of skin effects or perturbations from the drilling process, the signal towards the injection or observation well would be remarkably different. This effect has been studied by Butler [4] in confined aquifers and by Trinchero et al. [40] in leaky aquifers.

The lack of a proper understanding of point-to-point connectivity in a more quantitative manner has prevented its use in many field applications. In particular, proper understanding of connectivity together with a reliable characterization of the geological medium will benefit and improve available methods used to delineate capture zones or perimeter protection areas in heterogeneous aquifers. Still, a large number of publications have addressed the topic indirectly by means of ensembles averages of numerical Monte Carlo simulations [37,41,7,17,30]. 


\section{Motivation}

Pumping and tracer tests are two well-established techniques for aquifer characterization. Albeit real aquifers are heterogeneous, the interpretation of these field tests is frequently conducted using well-known homogeneous analytical solutions for convenience. In doing this, the effects of heterogeneity are somehow lumped into the estimated aquifer parameters, making them to intrinsically depend on the specific hydrodynamic processes and the interpretation method.

Employing these parameters in any realistic flow and solute transport model requires the fundamental understanding of the influence of heterogeneity, which sometimes can drastically modify the true physical meaning of the estimated parameters. This is the case for instance of $S_{\text {est }}$ estimated using the Cooper-Jacob method in pumping tests and $\phi_{\text {est }}$ estimated from the characteristic advective travel time of a solute tracer test. Being consistent with the fact that these parameters actually measure the travel velocity of the response signal between two points of the aquifer, in the same line of previous works [21,22], we consider $S_{\text {est }}$ and $\phi_{\text {est }}$ as indicators of flow and transport point-to-point connectivity, respectively. These indicators of connectivity based on state variables not only depend on whether two points of the aquifer are actually connected by geological lineaments/fractures but also include the interplay of the physical flow and/or transport processes causing $\phi_{\text {est }}$ to give not exactly the same information as $S_{\text {est }}$.

We note that these indicators strictly depend on the interpreta-
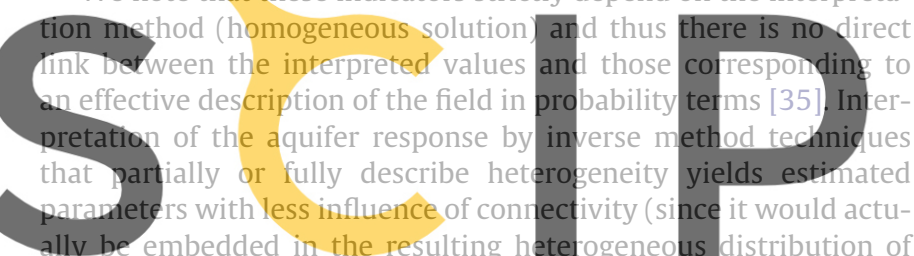

parameters). If heterogeneity is accurately described in detail then $\phi_{\text {ast }}$ leads to the true porosity of the system.

Register for free at https//WWW.scipedia.com to

\section{Field and laboratory observations}

\subsection{Pumping tests}

The hydraulic response of pumping tests has been frequently observed in the field to substantially vary with the observation location. To illustrate this, Fig. 1 shows the time-drawdown data

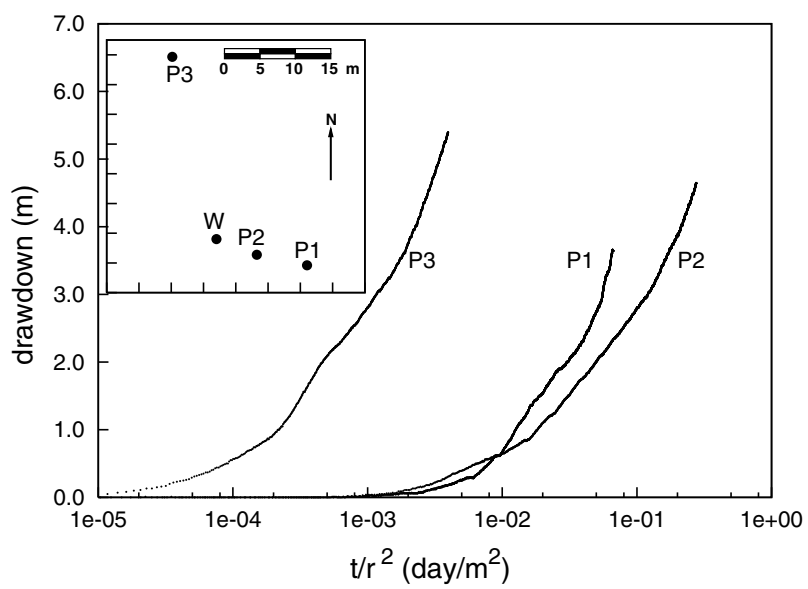

Fig. 1. Drawdown curves of a pumping test performed in a fractured media in Spain. In the small box it is represented the space location of the well, $\mathrm{W}$, and the three piezometers, P1, P2 and P3. In a homogeneous medium the three curves would collapse. of a pumping test performed in a fractured media in Southern Spain where the flow is mainly horizontal. Drawdown data were measured in three different piezometers and each timedrawdown curve was interpreted separately using the CooperJacob method. In short, this well-known method consists in plotting the drawdown versus log-time curve. The transmissivity, $T_{\text {est }}$, is obtained from the late-time slope of the drawdown curve, $m$, and the storage coefficient, $S_{\text {est }}$, is obtained from the time at which the projection line of the slope intercepts the zero drawdown axis, $t_{0}$

$T_{\text {est }}=0.183 \frac{Q}{m}$,

$S_{\mathrm{est}}=\frac{2.25 T_{\mathrm{est}} t_{0}}{r^{2}}$.

We note that while the late-time slope of the drawdown curves depicted in Fig. 1 is almost the same for the three piezometers, a large variability is observed in the hydraulic response, having P3 the fastest response (despite the point is located further away from the well). The corresponding estimated parameters are shown in Table 1. We emphasize that while $T_{\text {est }}$ is almost constant for all observations, $S_{\text {est }}$ varies up to two orders of magnitude. Meier et al. [26] attributed these large variations in $S_{\text {est }}$ to a good hydraulic connectivity between the pumping well $\mathrm{W}$ and piezometer P3 and a relatively bad hydraulic connectivity between the well and piezometers P1 and P2. These connectivity features are actually clearly visible in this particular site by mapping the geologic linea-

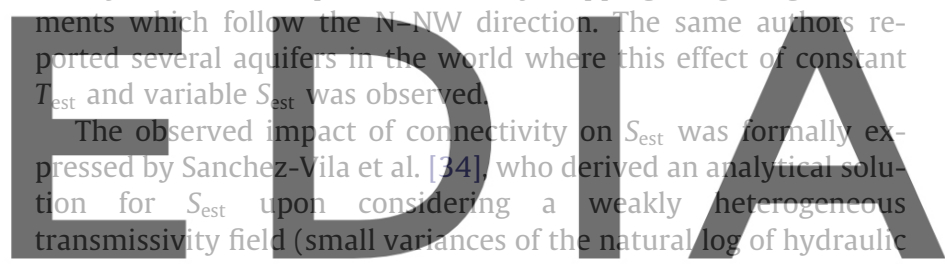
conductivity) and no boundary effects (infinite medium). Then, by truncation of an infinite series expansion, they found an approxi-

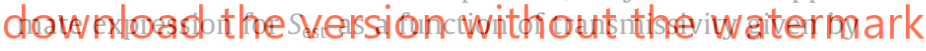

$S_{\text {est }}\left(r_{0}, \theta_{0}\right)=\operatorname{Sexp}\left(-\frac{1}{\pi} \int_{0}^{2 \pi} \int_{0}^{\infty} Y^{\prime}(\rho, \varphi) U\left(r_{0}, \theta_{0}, \rho, \varphi\right) \mathrm{d} \rho \mathrm{d} \varphi\right)$

where $S$ is the actual storage coefficient representative of the aquifer system (assumed constant), $Y^{\prime}(\mathbf{x})=\ln \left(T(\mathbf{x}) / T_{0}\right), T(\mathbf{x})$ is the point value of transmissivity at the $\mathbf{x}$ location, $T_{0}$ is the effective aquifer transmissivity, $(\rho, \varphi)$ are the polar coordinates centered at the pumping well, $\left(r_{0}, \theta_{0}\right)$ is the observation location in polar coordinates, and $U\left(r_{0}, \theta_{0}, \rho, \varphi\right)$ is a weighting function defined as

$U\left(r_{0}, \theta_{0}, \rho, \varphi\right)=-\frac{\rho-r_{0} \cos \left(\theta_{0}-\varphi\right)}{\left(\rho^{2}+r_{0}^{2}-2 \rho r_{0} \cos \left(\theta_{0}-\varphi\right)\right) \rho}$.

This expression not only agrees with field observations in that $S_{\text {est }}$ varies with the observation location, but further describes the dependency of $S_{\text {est }}$ on the transmissivity features by means of a weighted spatial integral over the entire space. From the shape of the weighting function, depicted in Fig. 2, we see that large transmissivity values (larger than $T_{0}$ ) located between the well and the observation point leads to $S_{\text {est }}$ smaller than $S$ and vice versa.

Table 1

Interpretation of the pumping test of Fig. 1 using the Cooper-Jacob method

\begin{tabular}{lll}
\hline & $T_{\text {est }}\left(\mathrm{m}^{2} /\right.$ day $)$ & $S_{\text {est }}$ \\
\hline P1 & $2 \times 10^{-1}$ & $4 \times 10^{-2}$ \\
P2 & $3 \times 10^{-1}$ & $2 \times 10^{-2}$ \\
P3 & $3 \times 10^{-1}$ & $3 \times 10^{-4}$ \\
\hline
\end{tabular}



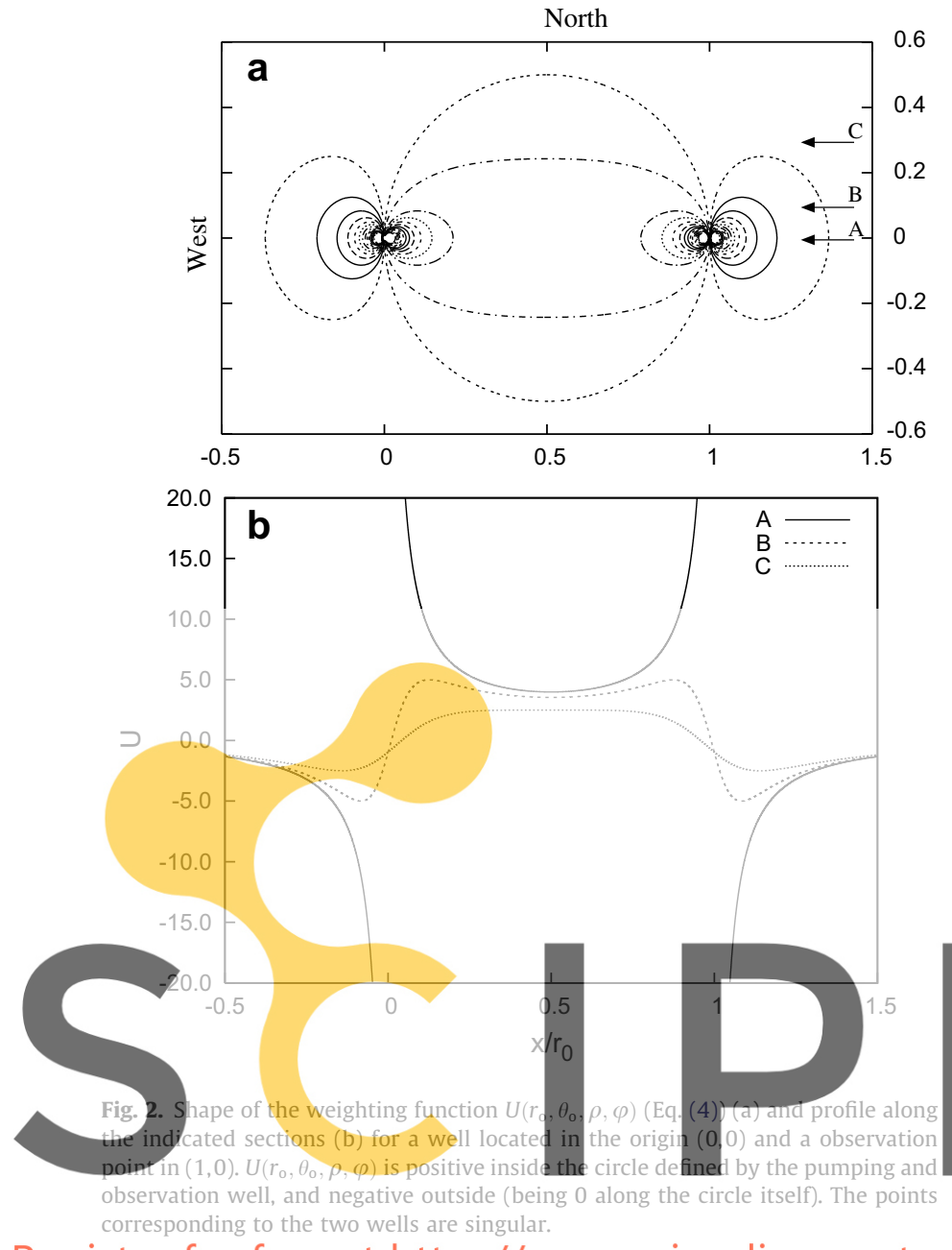

corresponding to the two wells are singular.
Register for free at https//www.scipedia.com to

\subsection{Laboratory tracer tests}

Field tracer tests constitute a valuable tool to estimate the governing parameters of solute transport in an aquifer. Known chemical species (tracers) are carefully added into the system and monitored with time and/or with space. Among all configurations, convergent-flow tracer tests are typically preferred for simplicity. In this case, once quasi steady-state flow conditions are established, the tracer is injected at a point located several meters away from a well that is pumped at a constant rate. Concentrations with time (breakthrough curve) are measured at the pumping well. Then, transport parameters are typically obtained by curve-fitting the tracer breakthrough curve with a known homogeneous transport model solution. Additionally, porosity estimates can also be determined from the advective travel time of the solute tracer, $t_{\mathrm{a}}$, as

$\phi_{\text {est }}=\frac{Q_{\mathrm{w}} t_{\mathrm{a}}}{\pi\left(r_{i}^{2}-r_{\mathrm{w}}^{2}\right)}$,

where $r_{i}$ is the radial distance between the pumping well and the tracer injection location, $r_{\mathrm{w}}$ is the well radius, and $Q_{\mathrm{w}}$ is the pumping rate per unit of thickness. Notice that with this definition $\phi_{\text {est }}$ and $t_{\mathrm{a}}$ are linearly related, so that they contain exactly the same amount of information. In general, $t_{\mathrm{a}}$ is estimated as the first normalized temporal moment of the breakthrough curve,

$t_{\mathrm{a}}=\frac{\int_{0}^{\infty} t C(t) \mathrm{d} t}{\int_{0}^{\infty} C(t) \mathrm{d} t}$.

Heterogeneity has been observed to largely influence the porosity estimates, $\phi_{\text {est }}$, obtained from (5). When there is a high $T$ path connecting both points, $\phi_{\text {est }}$ is lower than $\phi$; contrariwise, if a low permeable lineament is located in between preventing the tracer to arrive to the well, $\phi_{\text {est }}$ will be higher than $\phi$. We refer to these effects as transport point-to-point connectivity. Although these effects are routinely observed in the field, the analysis of connectivity based on field data is cumbersome because of a limited number of available tracer experiments and large uncertainties inherent to the depiction of the heterogeneous formation.

In this context, intermediate-scale laboratory tracer experiments performed in reconstrycted aquifers provide valuable infor-
mation to study the processes governing connectivity under well
controlled conditions. In the laboratory, good knowledge of the
physical systen and numerous tracer experiments can be feasibly
achieved. Fernandez-Garcia et fl. [11] investigated transport con-
nectivity in a reconstructed anisotrop c heterogenfous aquifer in
the laboratory. The physical system was created by packing together sand lenses of different hydraulic conductivities in a hori-

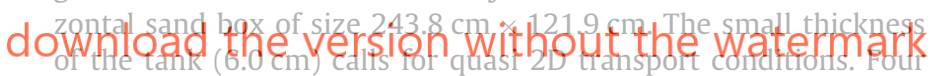
different clean sands, mostly dominated by silica minerals, were used. The distribution of the sand was deterministically generated following two premises: (1) greater length of sand lenses was assigned to the longitudinal direction (ratio between $1: 2$ and $1: 8$ ); and (2) nearby sands had similar hydraulic features. Fig. 3 depicts the spatial distribution of the different sands used in the experiments.

A constant head spill reservoir was connected to the sides of the sand box to provide constant head conditions at the external boundary of the aquifer domain. To create convergent-flow conditions, water was extracted from a centered well at a constant rate $(180 \mathrm{ml} / \mathrm{min})$. Once steady-state flow was reached, several

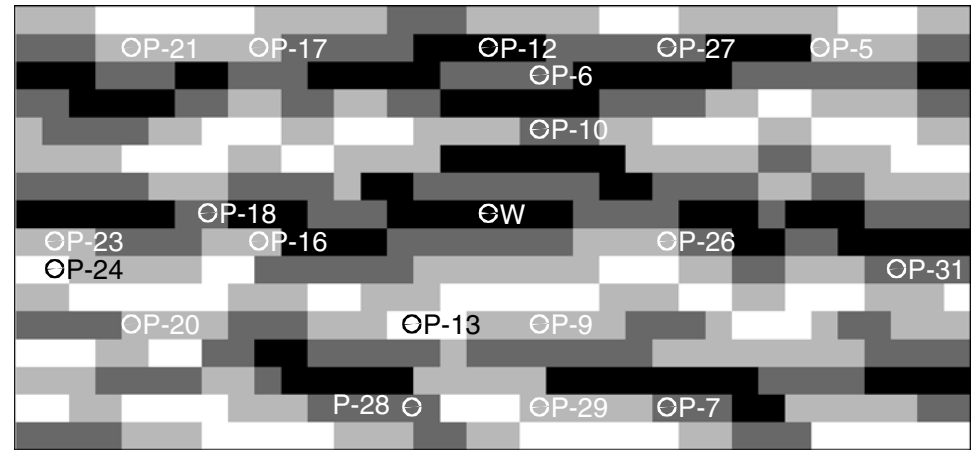

Sand 4

\section{Sand 3}

Sand 2

\section{Sand 1}

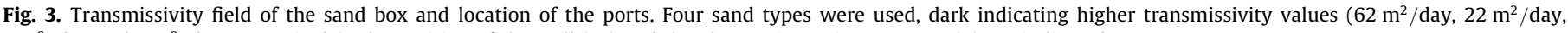
$7 \mathrm{~m}^{2} /$ day and $2 \mathrm{~m}^{2} /$ day respectively). The position of the well (W) and the observation points or ports (P) are indicated. 


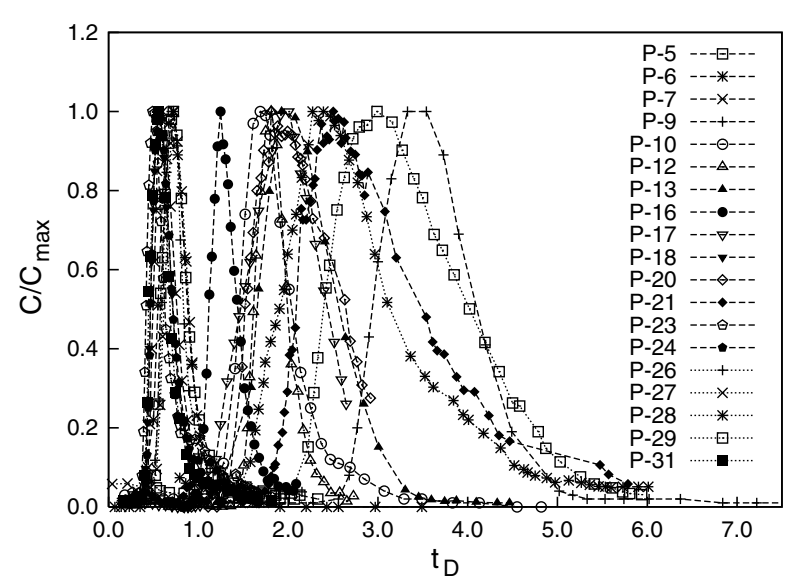

Fig. 4. Normalized breakthrough curves obtained at different injection location: $t_{D}$ is the dimensionless time $\left(t_{D}=O t / \pi r^{2} b \phi\right)$, where $O$ is the pumping rate, $r$ the distance from the well, $b$ the aquifer thickness and $\phi$ the actual porosity. In a homogeneous media all curves shouid superimpose.

forced-gradient tracer experiments were sequentially conducted by injecting a slug of a sodium bromide solution into the system (see Fig. 3 for the location of the injections). For each injection, enough total pore volumes were pumped out from the system to prevent any interference between tracer experiments. Corresponding bromide breakthrough curves are summarized in Fig. 4. More details are found in Fernandez-Garcia their results using a dimensionless tim differences that were observed relative 1 genequs porous medium. In the hom through curves should exhibit the same shape with a center of
gravity located at $t_{\mathrm{D}}=1.0$. The authors found that the mean travel time reflected in an individual breakthrough curve mostly depends on the particular hydraulic features existing between the pumping

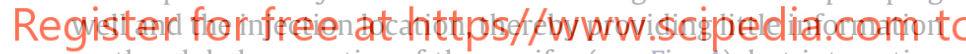
on the global properties of the aquifer (see Fig. 4), but interesting information regarding point-to-point (transport) connectivity.

\section{Analytical relationship between $\phi_{\text {est }}$ and $S_{\text {est }}$}

As stated in the previous section, the two connectivity indicators, $\phi_{\text {est }}$ and $S_{\text {est }}$, provide a different measure of connectivity. In this section we explore whether it is possible to relate them. In the following we consider a two-dimensional porous media under convergent-flow conditions induced by a pumping well in an otherwise static pressure aquifer system. Boundaries are considered to be located sufficiently far from the area of study so that their impact upon drawdown is negligible. Water is extracted at a constant rate and the flow system is considered at quasi-steady-state (drawdown gradients do not change in time).

Considering that the spatial variability of transmissivity occurring in all natural aquifers is the dominant variable responsible for the existence of preferential flow channels in the system, we view transmissivity as the only varying property in space. The storage coefficient $(S)$ and the effective porosity $(\phi)$ are assumed spatially constant in the analysis. This is a common workable assumption in stochastic subsurface hydrology since the spatial variability of $T$ is orders of magnitude larger than that of $S$ and $\phi$.

Transport point-to-point connectivity involves those physical properties by which the characteristic advective time between the pumping well and the observation location is enhanced or reduced relative to that of a homogenized equivalent system. Thus, we start by analyzing the advective travel time of a non-reactive mass particle, $t_{\mathrm{a}}$, injected instantaneously into the system,
$t_{\mathrm{a}}=\int_{\Gamma} \frac{\phi}{\|\mathbf{q}\|} \mathrm{d} s$

where $\Gamma$ is the trajectory of the injected particle, $\phi$ is the effective porosity (ratio of mobile aqueous phase volume to the total volume), and q is the Darcy's velocity. Assuming that the particle path line does not significantly deviate from the mean fluid motion we can parameterize (7) using a radial coordinates system centered at the pumping well

$t_{\mathrm{a}}\left(r_{i}, \theta_{i}\right)=-\phi \int_{r_{\mathrm{w}}}^{r_{i}} \frac{1}{q_{\mathrm{r}}(r, \theta(r))} \mathrm{d} r$

In (8) $\left(r_{i}, \theta_{i}\right)$ denotes the radial coordinates of the injection location, $r_{\mathrm{w}}$ is the pumping well radius, and $q_{\mathrm{r}}$ is the radial component of the Darcy's velocity

$q_{\mathrm{r}}(r, \theta)=-e^{Y} \frac{\partial h}{\partial r}$,

where $Y(\mathbf{x})=\ln T(\mathbf{x})$, and $h$ is the drawdown which is in turn governed by the following partial differential equation:

$\nabla Y(\mathbf{x}) \nabla h(\mathbf{x}, t)+\nabla^{2} h(\mathbf{x}, t)=e^{-Y} S \frac{\partial h(\mathbf{x}, t)}{\partial t}$.

Here, $S$ is the true storage coefficient of the aquifer (released pore-water per unit area of the aquifer and unit decline of the piezometric head), assumed also spatially constant. Eq. (10) is strictly valid only in confined aquifers, but it can be a valid approximation in unconfined aquifers whenever the drawdown is small with respect to the initial saturated thickness. In the presence of a
pumping well, the corresponding boundary and initial conditions
ate written as
$h(\mathbf{x}, t=0)=0$
$h(|\mathbf{x}| \rightarrow \infty, t)=0$
$\lim _{r \rightarrow r_{w}}\left(2 \pi r \frac{a h}{\partial r}\right)=Q_{w} e^{-Y_{0}}$

chowendoadhethenversionewithoutithesswatermark Quasi-steady-state conditions are achieved at large times. Since $Y(\mathbf{x})$ is randomly varying in space, it is in general not possible to obtain analytical solutions of $t_{\mathrm{a}}$ from (8). To overcome this probiem, we used perturbation theory to find an approximate solution. The approach starts by writing $t_{\mathrm{a}}$ and $h$ in an expansion series

$t_{\mathrm{a}}=t_{\mathrm{a}}^{(0)}+t_{\mathrm{a}}^{(1)}+t_{\mathrm{a}}^{(2)}+\cdots$,

$h=h^{(0)}+h^{(1)}+h^{(2)}+\cdots$

The leading terms $t_{\mathrm{a}}^{(0)}$ and $h^{(0)}$ are the solutions of two deterministic problems obtained by substituting the heterogeneous $Y$-field by an equivalent $\log$ transmissivity, $Y_{0}=\ln T_{0}$. The other terms, $t_{\mathrm{a}}^{(i)}$ and $h^{(i)}(i=1,2, \ldots)$, are solutions of stochastic partial differential equations describing the deviations from the homogeneous solution. These terms are of the order of some characteristic scalar value, usually taken as the standard deviation of the natural log of hydraulic conductivity, $\sigma_{Y}$. Thus for small $\sigma_{Y}$ the higher order terms, $t_{\mathrm{a}}^{(i)}$ and $h^{(i)}$, become successively less important. Keeping only the first two terms in the expansion, we write

$q_{\mathrm{r}}(\mathbf{x})=q_{0}(r)+q_{\mathrm{r}}^{\prime}(\mathbf{x})$

Taylor expansion around $\left(r, \theta_{i}\right)$ yields

$$
\begin{aligned}
t_{\mathrm{a}}\left(r_{i}, \theta_{i}\right)= & -\phi \int_{r_{\mathrm{w}}}^{r_{i}} \frac{1}{q_{0}(r)} \\
& \times\left(1-\frac{q_{\mathrm{r}}^{\prime}\left(r, \theta_{i}\right)}{q_{0}(r)}+\left(\frac{q_{\mathrm{r}}^{\prime}\left(r, \theta_{i}\right)}{q_{0}(r)}\right)^{2}+\frac{\partial q_{\mathrm{r}}^{\prime}\left(r, \theta_{i}\right)}{\partial \theta}\left(\theta-\theta_{i}\right)+\cdots\right) \mathrm{d} r .
\end{aligned}
$$


By setting

$Y(\mathbf{x})=Y_{0}+Y^{\prime}(\mathbf{x})$,

$Y_{0}$ being a representative mean value of $Y$ (e.g. the arithmetic mean), and introducing (15) into Darcy's law (9), and using the Taylor expansion of $\exp \left(Y^{\prime}\right)$ we obtain

$$
\begin{aligned}
q_{\mathrm{r}}(r, \theta) & =-T_{0}\left(1+Y^{\prime}+\frac{Y^{\prime 2}}{2}+\cdots\right) \frac{\partial}{\partial r}\left(h^{(0)}+h^{(1)}+\cdots\right) \\
& =-T_{0}\left(\frac{\partial h^{(0)}(r)}{\partial r}+\frac{\partial h^{(0)}(r)}{\partial r} Y^{\prime}(r, \theta)+\frac{\partial h^{(1)}(r, \theta)}{\partial r}\right)+\cdots
\end{aligned}
$$

The first term on the right-hand side of (19) is $q_{0}(r)$, which is the solution for a radial flow system in homogeneous medium,

$q_{0}(r)=-T_{0} \frac{\partial h^{(0)}(r)}{\partial r}=-\left(\frac{Q_{w}}{2 \pi}\right) \frac{1}{r}$.

Subtracting the homogeneous solution $q_{0}(r)$ from $q_{r}(r, \theta)$ we obtain $q_{r}^{\prime}(r, \theta)=q_{0}(r) Y^{\prime}(r, \theta)-T_{0} \frac{\partial h^{(1)}(r, \theta)}{\partial r}+\cdots \cdot$

Thus, the Darcy's velocity deviations from the equivalent homogeneous solution depend on the transmissivity field and also on the space distribution of the piezometric head. The solution of the terms $h^{(i)}$ can be obtained using an iterative procedure. We refer to the work of Sanchez-Vila et al. [34] for the actual derivation of the solution. Considering a small well diameter $\left(r_{w} \rightarrow 0\right)$, the first
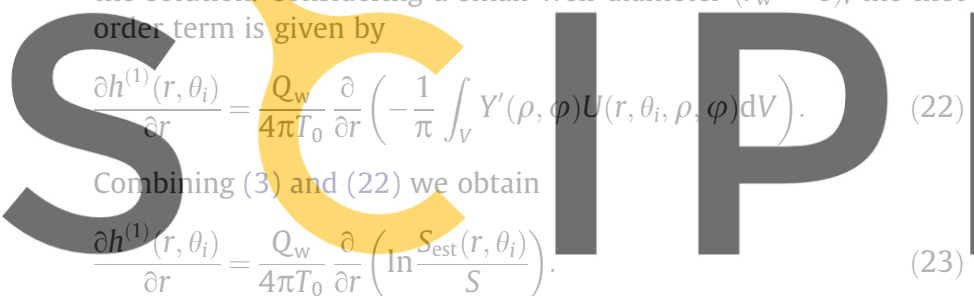

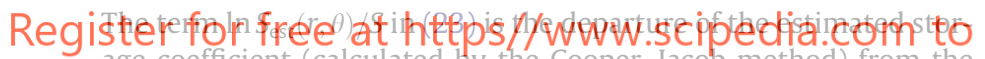 age coefficient (calculated by the Cooper-Jacob method) from the actual coefficient $S$.}

Considering again $r_{\mathrm{w}} \rightarrow 0$, we derive the relationship between the characteristic advective travel time, $t_{\mathrm{a}}$, and the estimated storage coefficient, $S_{\text {est }}$, by simply substituting (23) and (20), (21) into (17) and integrating by parts

$t_{\mathrm{a}}\left(r_{i}, \theta_{i}\right)=\frac{\phi \pi r_{i}^{2}}{Q_{\mathrm{w}}}\left(1-\int_{0}^{r_{i}} \frac{2 r}{r_{i}^{2}}\left\{Y^{\prime}\left(r, \theta_{i}\right)-\ln \frac{S_{\text {est }}\left(r, \theta_{i}\right)}{S_{\text {est }}\left(r_{i}, \theta_{i}\right)}\right\} \mathrm{d} r\right)$.

The term $\ln S_{\text {est }}\left(r, \theta_{i}\right) / S_{\text {est }}\left(r_{i}, \theta_{i}\right)$ in (24) denotes the departure of the estimated storage coefficient at a given point $\left(r, \theta_{i}\right)$ located between the injection and the pumping well with respect to the estimated storage coefficient at the injection location $\left(r_{i}, \theta_{i}\right)$.

The series expansion in (24) is truncated at the first-order in $\sigma_{Y}$. In an attempt to overcome the latter truncation, we view (24) as the first two terms of an exponential expansion and we generalized the final solution as

$t_{\mathrm{a}}\left(r_{i}, \theta_{i}\right)=\frac{\phi \pi r_{i}^{2}}{Q_{\mathrm{w}}} \exp \left(-\int_{0}^{r_{i}} \frac{2 r}{r_{i}^{2}}\left\{Y^{\prime}\left(r, \theta_{i}\right)-\ln \frac{S_{\text {est }}\left(r, \theta_{i}\right)}{S_{\text {est }}\left(r_{i}, \theta_{i}\right)}\right\} \mathrm{d} r\right)$.

This exponentiation is a widely used procedure in stochastic subsurface hydrology (e.g., [15]). Otherwise, the estimation of $t_{\mathrm{a}}$ through (24) may yield negative values, which are not physically correct. Recalling (5), this last expression can be written in terms of the estimated porosity obtained from interpreting concentration breakthrough curves of convergent-flow tracer tests

$\phi_{\text {est }}\left(r_{i}, \theta_{i}\right)=\phi \exp \left(-\int_{0}^{r_{i}} \frac{2 r}{r_{i}^{2}}\left\{Y^{\prime}\left(r, \theta_{i}\right)-\ln \frac{S_{\text {est }}\left(r, \theta_{i}\right)}{S_{\text {est }}\left(r_{i}, \theta_{i}\right)}\right\} \mathrm{d} r\right)$
Here, the inverse of $\phi_{\text {est }}$ is viewed as an indicator of transport pointto-point connectivity because it essentially depends upon travel time, which is directly controlled by connectivity. In the homogeneous case, $Y^{\prime}=0, \ln S_{\text {est }}=S$, and thus $\phi_{\text {est }}=\phi$. In agreement with field and lab observations, (26) demonstrates that in heterogeneous media the estimated porosity $\phi_{\text {est }}$ from a tracer test depends not only on $\phi$ (actual porosity), but also on $Y^{\prime}$ and $S_{\text {est }}$ along the line connecting the pumping and the injection wells. Thus, $\phi_{\text {est }}$ is not a physical parameter but rather a value coming from the test interpretation method.

In (26) we can view $2 r / r_{i}^{2}$ as a weighting function, increasing linearly from the well to the injection point. Close to the well, the information provided by $Y^{\prime}$ and $S_{\text {est }}$ does not provide any additional information on $\phi_{\text {est }}$, since the weighting function is almost null. This is logical, since the travel time to the well is not affected by the actual measurements taken at the well location [32]. On the contrary, largest weights are given to the values corresponding to points located close to the injection location. It should also be noted that $S_{\text {est }}$ in turn depends on a spatial average of $Y^{\prime}$ that

\section{North}

a
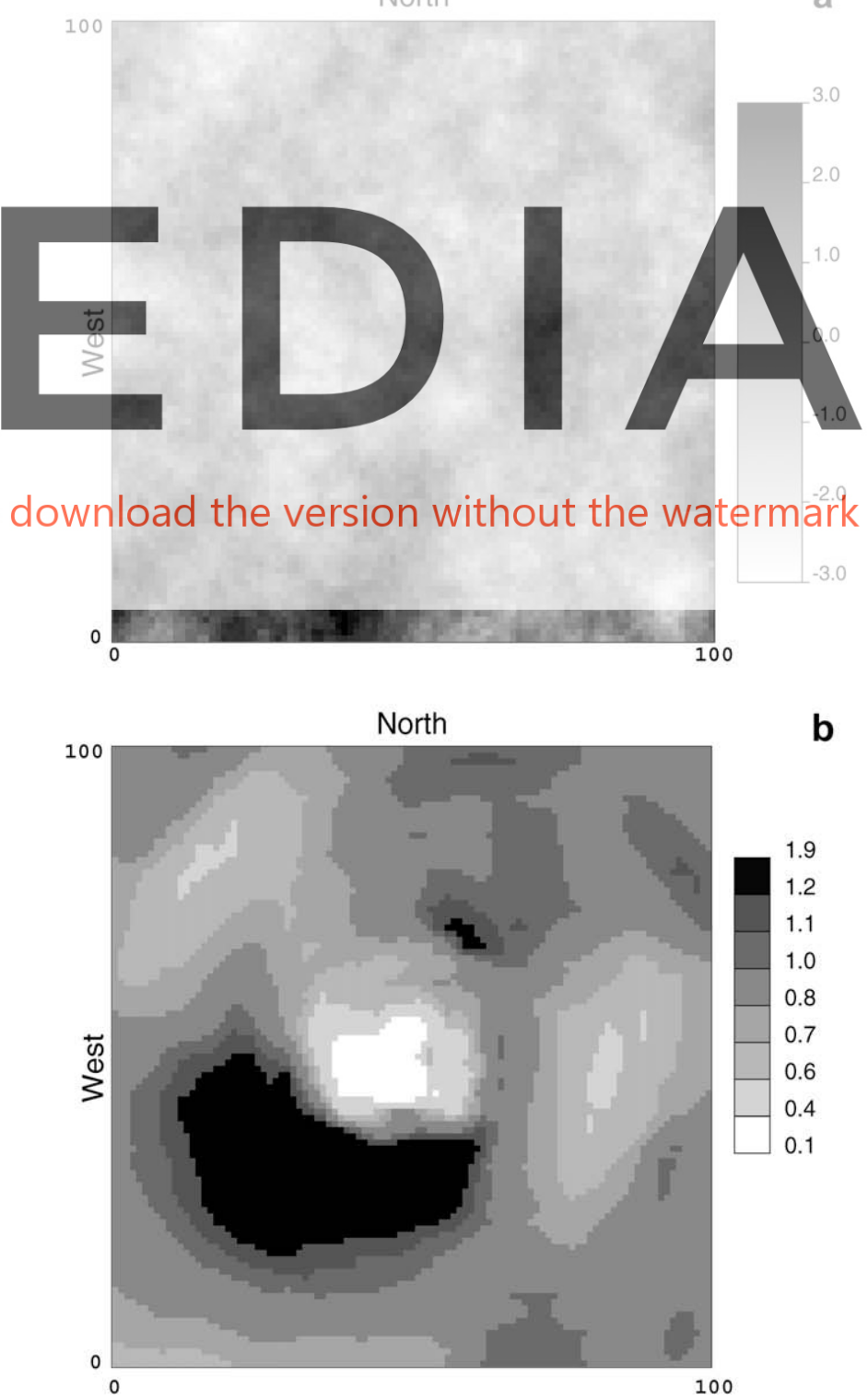

Fig. 5. Natural logarithm of the transmissivity field (a) and map of $S_{\text {est }} / S$ for the given aquifer (b), modified from Sanchez-Vila et al. [34]. Each map is representative of a subdomain of $101 \times 101$ cells centered around the well. 
extends throughout the entire system (Eq. (3)) and is characterized by a different weighting function, $U$ (Eq. (4)). This function is maximum along the line defined by the pumping and the observation wells, and largely weights the region nearby the two wells [34].

The final $\phi_{\text {est }} / \phi$ outcome is the balance of two processes. Whenever the transmissivity values along the particle path are larger than $T_{0}$ (equivalent field transmissivity), $Y^{\prime}>0$ and the first term in (26) tends to yield $\phi_{\text {est }}$ values below the representative value of porosity, $\phi$. This term directly depends on the transmissivity features between the pumping well and the injection location but does not include direct information on the hydraulic head response induced by pumping. All this information is provided by the second term in (26), that expresses that whenever the hydraulic response along the particle path are faster (small $S_{\text {est }}$ ) than that of the injection location, $\left(\ln S_{\text {est }}\left(r, \theta_{i}\right) / S_{\text {est }}\left(r_{i}, \theta_{i}\right)<0\right)$, the second term also tends to decrease $\phi_{\text {est }}$, and thus increase transport connectivity.

\section{Computational investigations}

5.1. Exploring the applicability of the analytical solution

The robustness of the analytical solution and the relative importance of the variables involved in (26) is examined in this section.

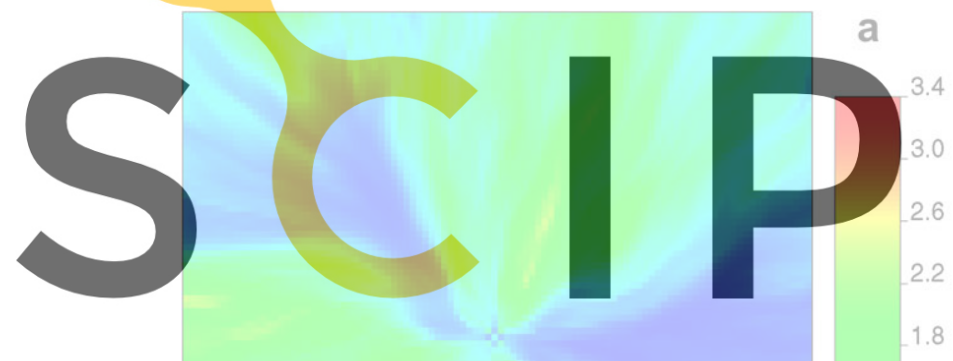

Register for free at https//www.scipedia.com.to
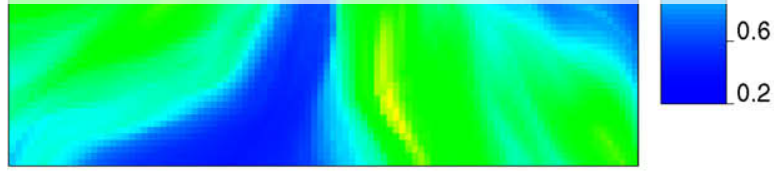

0.6
0.2
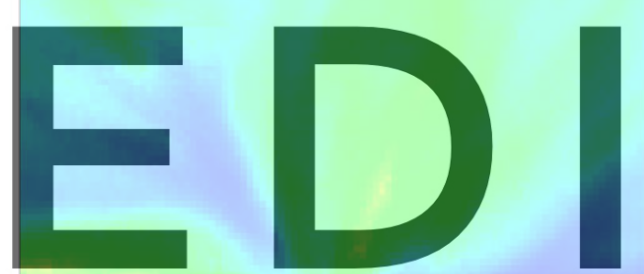

b

spherical variogram model with zero mean, unit variance, and integral scale of $10 \mathrm{~m}$.

An abstraction well was located at the center of the domain with prescribed flow rate, whereas zero drawdown was fixed at the external boundaries. The domain was artificially enlarged to avoid boundary effects. In this context, Sanchez-Vila et al. [34] numerically calculated the spatial variability of $S_{\text {est }}$ estimates by simulating the drawdown curves for all nodes of the corresponding groundwater flow model. Their results are shown in Fig. 5b for comparative purposes.

Here, we generate point-to-point transport connectivity maps by means of the indicator $\phi_{\text {est }}(\mathbf{x})$. Each $\phi_{\text {est }}(\mathbf{x})$ value is obtained by injecting a slug of mass into the system (at the $\mathbf{x}$ location), solving the corresponding transport problem and then using (5). The process was repeated for each grid cell in the domain.

First, we simulated steady-state flow in this setup using the finite difference code MODFLOW2000 [19]. The resulting velocity
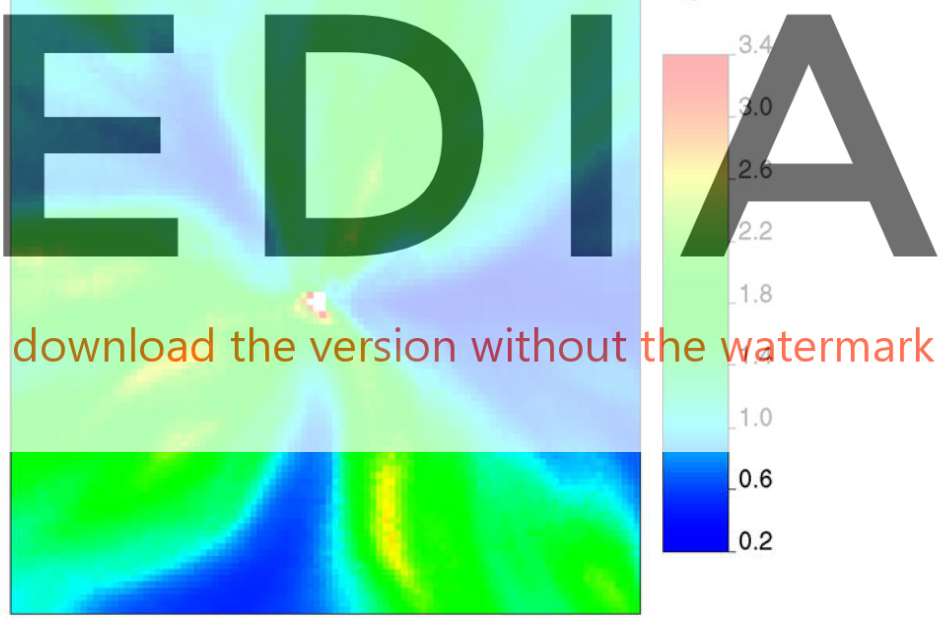
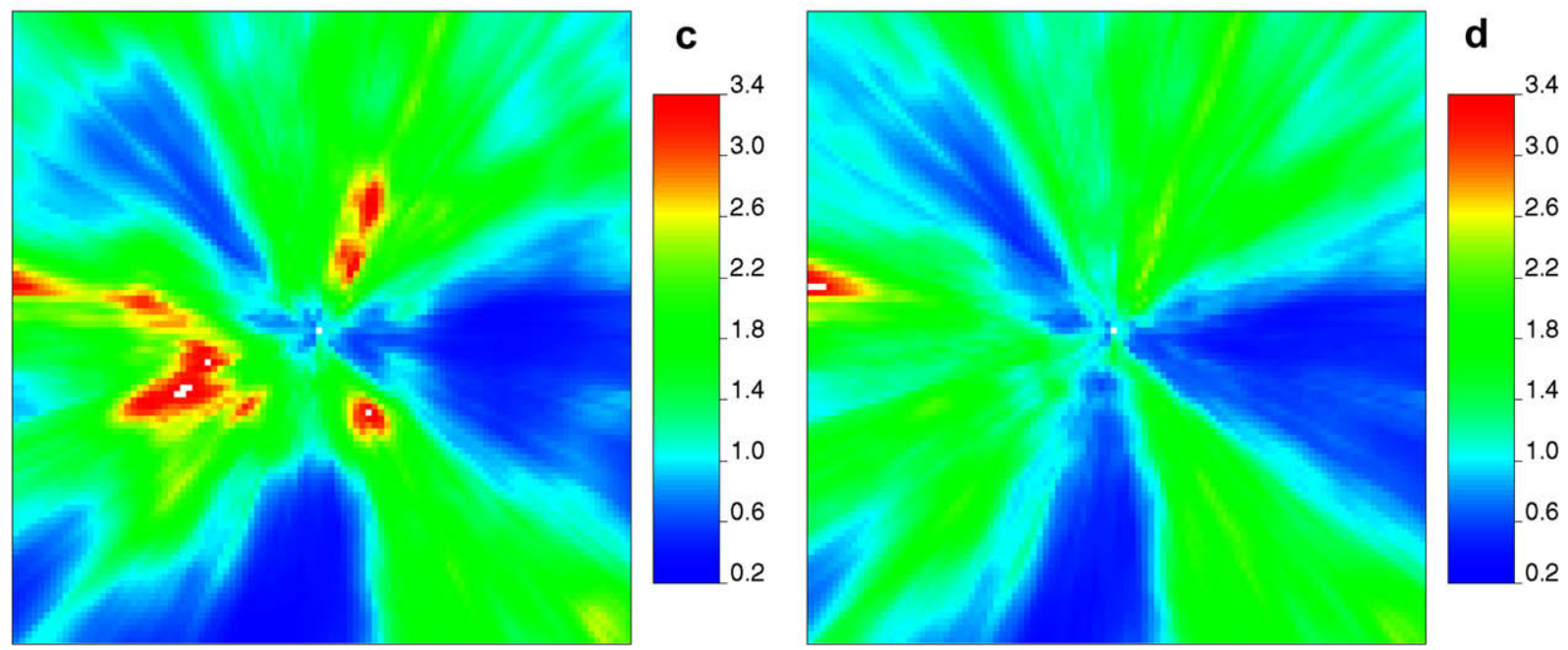

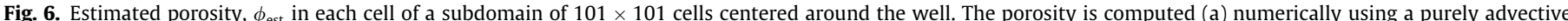

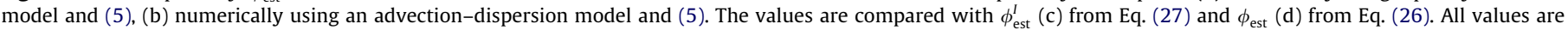
normalized by $\phi$. 
field was used to simulate solute transport, which is solved using a random walk particle tracking code, RW3D [12]. For each injection, the characteristic advective travel time was simulated in two ways: (a) using a purely advective model in which only a single particle is injected at the centroid of the grid cell to calculate $t_{\mathrm{a}}$ through (7); and (b) using and advective-dispersive model with 100 particles initially uniformly distributed within each cell. In this case, the characteristic advective travel time was estimated by the mean value of all the particle travel times recorded at the pumping well. The longitudinal and transverse dispersivity were set to $1 \mathrm{~m}$ and $0.1 \mathrm{~m}$, respectively.

Fig. 6a and b shows the maps of $\phi_{\text {est }}(\mathbf{x})$ for the two different conceptual transport models. We see that connectivity patterns were not substantially changed when including local dispersion processes into the transport model. This indicated that in this particular case, i.e., forced-gradient conditions induced by a pumping
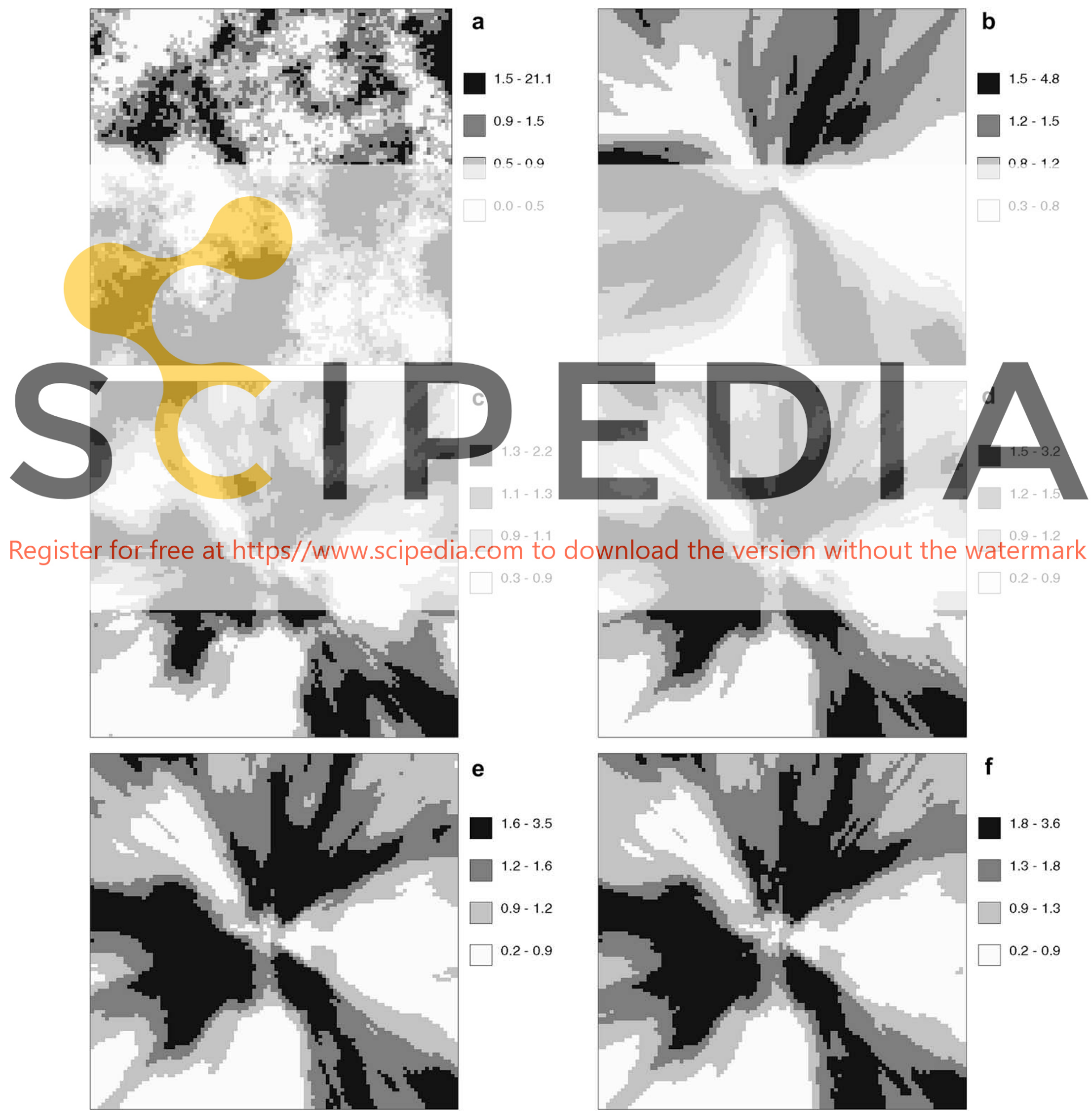

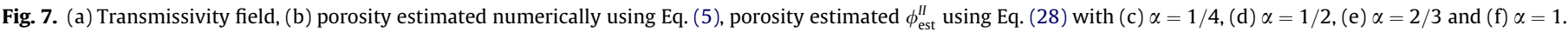
Each map is representative of a subdomain of $101 \times 101$ cells centered around the well. 
well in a continuous porous medium, point-to-point connectivity patterns measured by the advective travel time did not inherit the same stability problems as those observed for aquifers with high conductivity contrasts and under non-convergent-flow conditions [42].

In order to evaluate the relative importance of the different processes governing (26), we used a new estimate for $\phi$ given by neglecting all the terms depending on $S_{\text {est }}$ in (26)

$\phi_{\text {est }}^{I}\left(r_{i}, \theta_{i}\right)=\phi \exp \left(-\int_{0}^{r_{i}} \frac{2 r}{r_{i}^{2}} Y^{\prime}\left(r, \theta_{i}\right) \mathrm{d} r\right)$

Then, we compare the results given by (27) with those obtained from the numerical simulation of $\phi_{\text {est }}(\mathbf{x})$. The approximate solution (27) was computed considering $Y^{\prime}(\mathbf{x})=\ln T(x) / T_{\mathrm{g}}$. Comparison of Fig. $6 \mathrm{a}$ and b with $\mathrm{c}$ shows that $\phi_{\text {est }}^{I}(\mathbf{x})$ can still capture those zones well connected (low $\phi_{\text {est }}$ values) with the pumping well, although the size of the well connected zone to the south is slightly overestimated. The poorly connected zones (high $\phi_{\text {est }}$ values) are still delineated by $\phi_{\text {est }}^{I}(\mathbf{x})$, although in this case the size and the degree of connectivity is overestimated.

The complete analytical solution (26) incorporates not only the values of $Y^{\prime}(\mathbf{x})=\ln T(x) / T_{\mathrm{g}}$ but also the hydraulic response of the system through $S_{\text {est }}(\mathbf{x})$ (second term) whose values are borrowed from those numerically computed by Sanchez-Vila et al. [34] (Fig. 5b). Comparison of Fig. 6a and b with Fig. 6d illustrates that the $S_{\text {est }}(\mathbf{x})$ term improves the delineation of zones of low to moderate connectivity while has a minor impact on the depiction of the

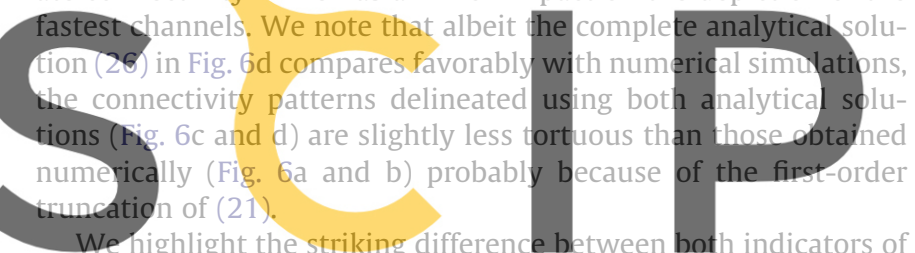
point-to-point connectivity: $\phi_{\text {est }}(\mathbf{x})$ (transport connectivity) and $S_{\text {est }}(\mathbf{x}$ ) (flow connectivity),(compare Figs. 6d and 5b, respectively).

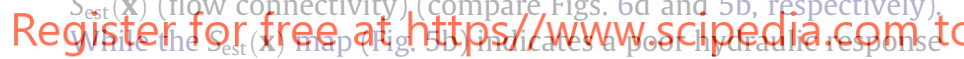

(black pixels) in the southern part of the domain, $\phi_{\text {est }}(\mathbf{x})$ is still well connected with the pumping well since large values of transmissivity are encountered in that region (Fig. 6d).

Eq. (27) relates the transmissivity values located along the line between the pumping well and the injection point to the estimated porosity. These transmissivity values are averaged by a linear weighting function that ranges from $2 / r_{i}$ at the injection location to zero at the well. This means that the influence of the $T$-values decreases as the particle travels towards the well where extreme gradients occur. From this consideration, it is worthwhile to assess the reliability of (27) when only a part of the $T$-values is used. This is achieved by examining the following approximation of $\phi_{\text {est }}(\mathbf{x})$ :

$\phi_{\text {est }}^{I I}\left(r_{i}, \theta_{i}\right) \approx \phi \exp \left(-\int_{r_{i}-\alpha r_{i}}^{r_{i}} \frac{2 r}{r_{i}^{2}} Y^{\prime}\left(r, \theta_{i}\right) \mathrm{d} r\right)$

where $\alpha$ is a constant ranging from 0 to 1 . Here, for simplicity, we divided the results in quartiles that could correspond to classes of connectivity (low, moderate, good and high). Fig. 7 shows the numerical simulation of (28) when $\alpha=1 / 4,1 / 2,2 / 3,1$. Using the information contained in only one-fourth of path line, i.e. $\alpha=1 / 4$, the approximation is still strongly influenced by the transmissivity at the injection location. This is the reason for Fig. 7c to have a similar shape as the transmissivity field (Fig. 7a). Using $\alpha=1 / 2$ the approximation is able to capture three well connected zones with the pumping well (east, north-west and south-west). Zones poorly connected are not so well delineated, mainly because (28) does not include the term in (26) involving the $S_{\text {est }}$ values. The use of

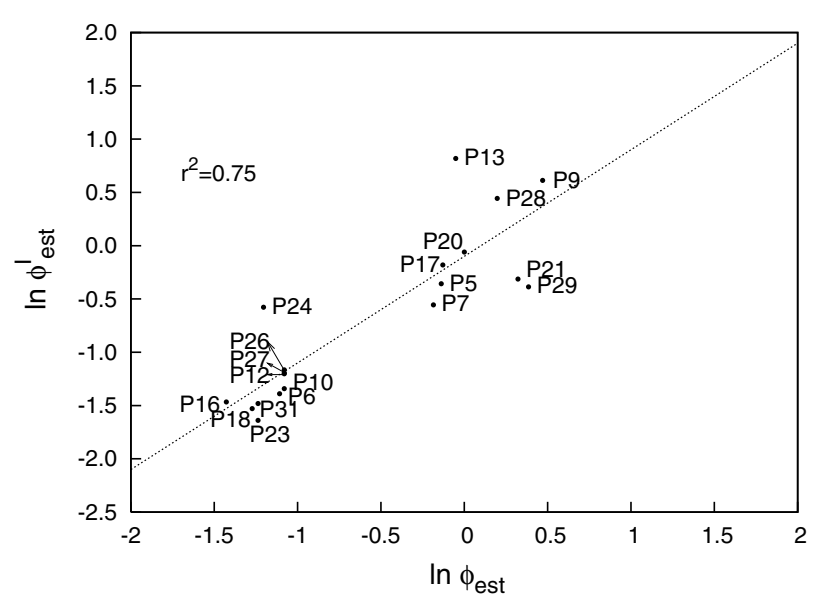

Fig. 8. Natural logarithm of the estimated porosity from laboratory, $\ln \phi_{\text {est }}$, versus the natural logarithm of the transmissivity estimated using Eq. (27) ( $\ln \phi_{\text {est }}^{I}$ ). The linear trendline and the correlation coefficient $\left(r^{2}\right)$ are indicated.

the information located in the last half of the line, (that are included using $\alpha=2 / 3$ and $\alpha=1$ ) leads to a very small improvement in the results (Fig. 7e and f).

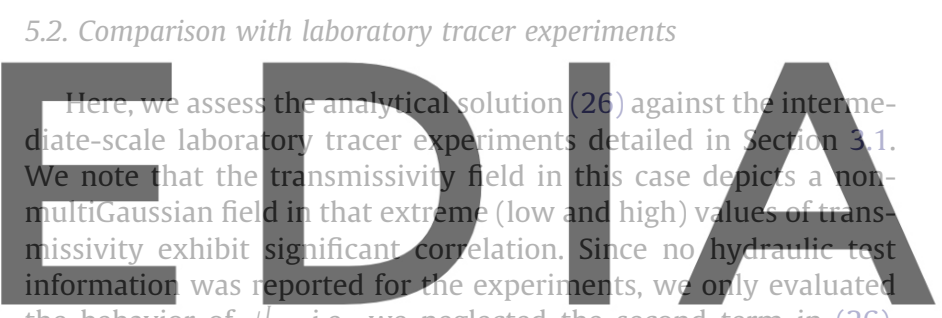

the behavior of $\phi_{\text {est }}^{t}$, i.e., we neglected the second term in (26), whose influence we showed to be limited to the delineation of

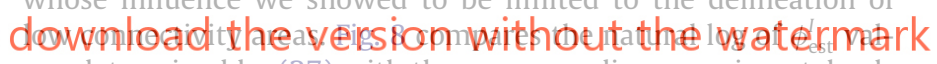
ues determined by (27) with the corresponding experimental values. The true porosity of the system used in (27) was obtained gravimetricaliy from core samples and is $\phi=0.44$ [11]. The equivalent transmissivity, $T_{0}$, was set equal to the arithmetic mean $\left(21 \mathrm{~m}^{2} /\right.$ day $)$. This value was determined by best fitting the experimental travel times, $\phi_{\text {est }}$, with their corresponding values. $T_{0}$ is close to the arithmetic mean probably because the aquifer system is well stratified. The results are shown in Fig. 8 and give a satisfactory linear correlation coefficient $\left(r^{2}=0.75\right)$ between the analytical solution and the experimental data. It must be noted that the calibration of $T_{0}$ would only affect the $y$-intercept of the regression line, having no influence in the linear correlation coefficient.

The comparison with the analytical solution (Fig. 8) is less accurate for ports P13 and P24. Based on the lab estimated porosity values, we note that these ports are located within, or close to, areas of poor point-to-point connectivity, which is consistent with the fact that $\phi_{\text {est }}^{I}$ overestimates $\phi_{\text {est }}$ in poorly connected areas (Section 5.1). The poor agreement observed in P21 and P29 can be attributed to boundary effects since both ports are located close to the limits of the domain.

\section{An application: delineation of capture zones}

The definition of protected areas, where anthropogenic activities are limited, is a key issue for the safeguard of water resources. Being economically and socially unfeasible to protect the entire aquifer, protection actions typically focus on small areas around the abstraction wells. As an example, the European Water Framework Directive requires the establishment of safeguard zones leav- 


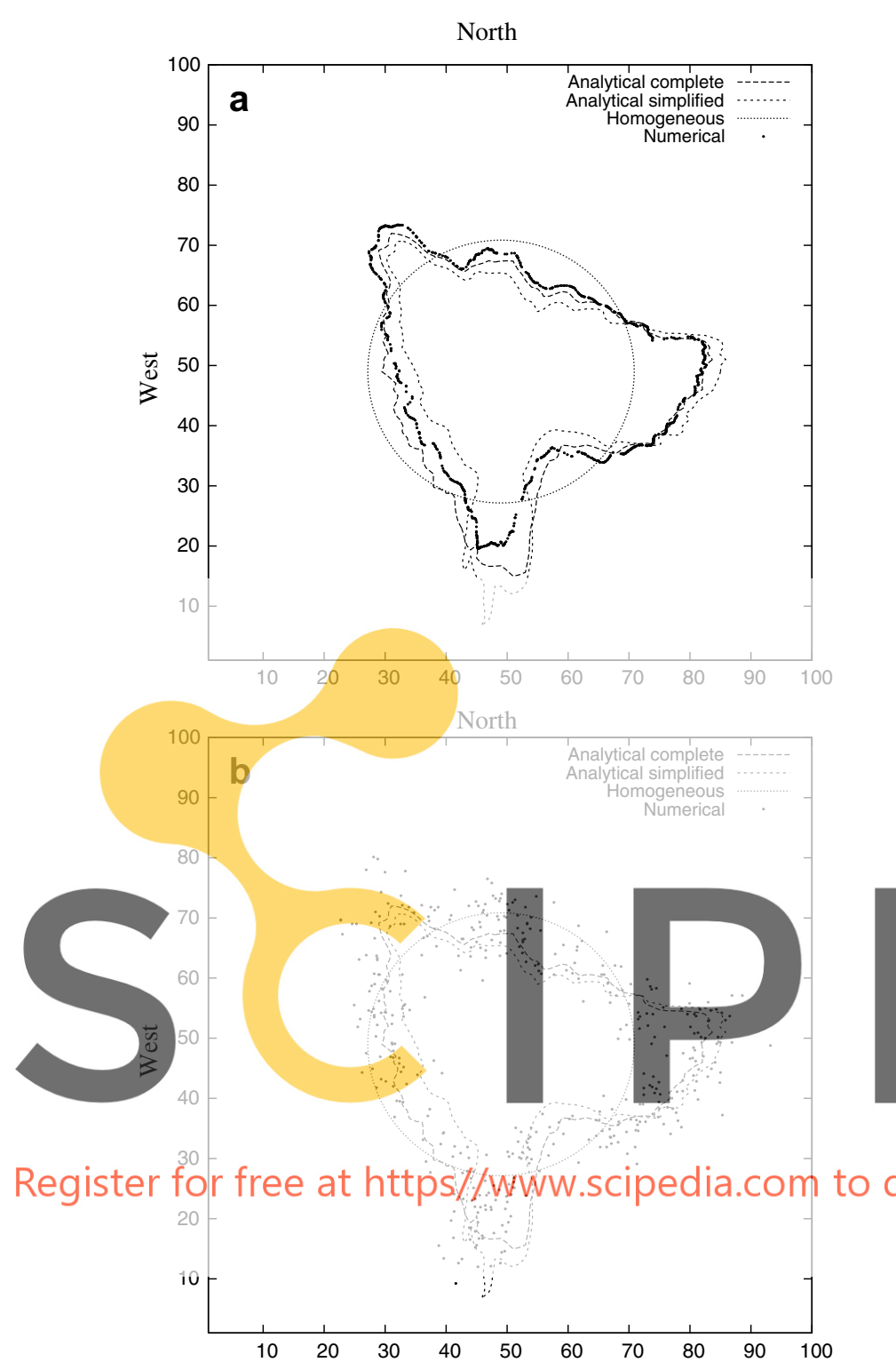

Fig. 9. Identification of the 50 days travel time capture zone from a well located at $(50,50)$. The numerical results have been obtained with backward simulations using (a) a purely advective model and (b) an advection-dispersion model. The analytical complete and simplified solutions refer to Eq. (26) and (27) respectively.

ing to the discretion of the member state the methodology to identify these areas. Nonetheless, in the guidance document on groundwater in drinking water protection areas [6], it is explicitly mentioned the 'time of travel to the abstraction' as a factor influencing the size and the shape of the capture zone. In this context, the impact of point-to-point transport connectivity on the identification of capture zones is of paramount importance. The mathematical framework and companion numerical simulations presented here give insight into the concept of transport connectivity which can be used to improve standard methodologies devoted to the delineation of capture zones.

To illustrate this, we use the analytical and simplified solutions (26) and (27) to delineate the protection perimeter around the abstraction well used in the synthetic aquifer of Section 5.

The protection perimeter was defined so that any accidental spill in a point located out of the perimeter will take more than 50 days to reach the pumping well. We adopted the same param- eters as those given in Section 5 , having a pumping rate of $Q_{\mathrm{w}}=30 \mathrm{~m}^{3} / \mathrm{m}$ day.

The capture zone obtained using the analytical solution was compared with numerical backward simulations. One thousand particles were injected at the well and backtracked to compute their position after 50 days. Two conceptual transport models were used. A purely advective transport model and an advective-dispersive model with longitudinal and transverse dispersivity set to $1 \mathrm{~m}$ and $0.1 \mathrm{~m}$, respectively.

Fig. 9a show the result of the purely advective model and clearly exhibits that the analytical solutions closely follow the numerical simulation results, thus capturing the most important connectivity features. For comparison purposes, these results are also contrasted with those associated with an equivalent homogeneous medium obtained by substituting the heterogeneous $T$-field by a homogeneous one. We see that the extension of the capture zone stretches over or falls inside the homogeneous perimeter in those parts of the domain with high and low connectivity, respectively. Thus, disregarding the role of connectivity induces a bias in the delineation of the capture zone in such a way that the size of well connected areas with the pumping well is underestimated. In agreement with Section 5, the simplified analytical solutions (27) depicted in Fig. 9 tends to overestimate the size and the degree of poorly connected zones. Highly connected areas are slightly overestimated with the exception of the northern zone (slightly underestimated).

The thousand particles of Fig. 9b show the backward location

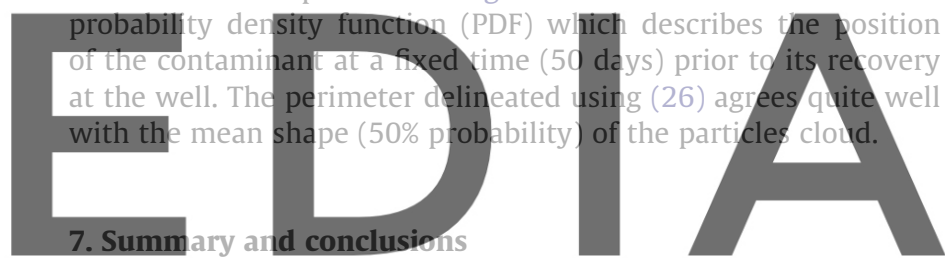

We present an explicit mathematical framework that assesses

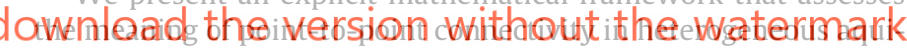
fers through the study of two commonly used indicators of connectivity ( $S_{\text {est }}$ and $\left.\phi_{\text {est }}\right)$. The analysis focuses on the impact of connectivity features on solute transport behavior under a radially convergent-flow system. Our analysis leads to the following main conclusions:

1. We have theoretically demonstrated that the estimated porosity, $\phi_{\text {est }}$, obtained from field tracer tests is an indicator of point-to-point transport connectivity rather than related to the total void volume of the aquifer. When the pumping well is hydraulically well/badly connected with a given location, $\phi_{\text {est }}$ takes values below/above the representative porosity of the aquifer.

2. This indicator of connectivity, $\phi_{\text {est }}$, involves two terms. The first term depends on the transmissivity values located along the flow path line such that large transmissivity values, i.e., larger than the equivalent homogeneous transmissivity of the aquifer, leads to estimates of $\phi_{\text {est }}$ smaller than $\phi$ and vice versa. The second term incorporates the hydraulic response of the aquifer (flow connectivity) induced by pumping, which is measured by the estimated storage coefficient, $S_{\text {est }}$ (calculated by the Cooper-Jacob method) defined by the observation point moving along the same path line. Point-to-point transport connectivity gets amplified/reduced when the time of the hydraulic response along the flow path line decreases/increases in the direction of flow. Both terms are weighted by a function that increases linearly with the radial distance from the pumping well. Thus, transport point-to-point connectivity is largely influenced by 
the aquifer properties close to the injection location, while transmissivity or $S_{\text {est }}$ data at points located nearby the pumping well provide no information on $\phi_{\text {est }}$.

3. We have theoretically demonstrated that the underlying processes governing transport connectivity are distinct from those involved in flow connectivity. The indicator of flow connectivity $S_{\text {est }}$ can be viewed as a weighted averaged over the entire domain, while the indicator of transport connectivity $\phi_{\text {est }}$ is a weighted averaged along the flow path line. Moreover, the transport weighting function (proportional to the radial distance) is remarkably different from that of flow connectivity, the latter assigning large weights to all the points located between the observation and the pumping well.

4. Comparison of the analytical solution of $\phi_{\text {est }}$ with numerical simulations performed in a given synthetic heterogeneous aquifer show that our mathematical framework is able to delineate low and high connectivity patterns with a good approximation in moderately heterogeneous aquifers. In particular, we found that the relative contribution of the hydraulic response $\left(S_{\text {est }}\right)$ to transport connectivity $\left(\phi_{\text {est }}\right)$ is minor, causing only a slight overestimation of low connectivity patterns. It is worthwhile to note that the analytical solution is obtained from a truncated perturbation expansion. Its accuracy in highly heterogeneous media should be explored in the future.

5. Delineation of point-to-point transport connectivity requires a limited amount of information since it mostly depends only on the transmissivity point values along the flow path line (minor contribution of $S_{\text {est }}$ ), being redundant the information nearby the well because of the shape of the transport weighting function.

6. The analytical solution of $\phi_{\text {est }}$ (27) was successfully contrasted with laboratory tracer experiments conducted in a reconstructed heterogeneous anisotropic medium with high correlation of extreme values (non-multiGaussian patterns). Results were consistent with our previous numerical observations. Well connected features were successfully captured by the analytical solution while the size and degree of low connectivity patterns were slightly overestimated.

7. The role of connectivity is seen to be of paramount importance for the delineation of protection areas (i.e. capture zones). Areas well connected with the pumping well exhibited protection perimeters that stretched beyond the equivalent homogeneous solution, i.e., the perimeter obtained considering a homogeneous aquifer. In this context, we present a novel simplified procedure to estimate the protection area of an abstraction well while honoring connectivity patterns. The methodology was successfully applied to a synthetic heterogeneous aquifer.

\section{Acknowledgements}

The authors acknowledge the financial support provided by ENRESA, by the Agència de Gestió d'Ajuts Universitaris i de Recerca of the Catalan Government and the Spanish CICYT (Project PARATODO). The authors also want to thank Jesus Carrera, Roger Beckie and two anonymous reviewers for their very helpful comments.

\section{References}

[1] Alcolea A, Castro E, Barbieri M, Carrera J, Bea S. Inverse modeling of coastal aquifers using tidal response and hydraulic tests. Ground Water 2007;45(6):711-22.

[2] Berkowitz B. Analysis of fracture network connectivity using percolation theory. Math Geol 1995;27(4):467-83.

[3] Bour O, Davy P. Connectivity of random fault networks following a power law fault length distribution. Water Resour Res 1997;33(7):1567-83.

[4] Butler JJ. Pumping tests in nonuniform aquifersthe radially symmetric case. J Hydrol 1988;101(1-4):15-30.
[5] Caers J, Zhang T. Multiple-point geostatistics: a quantitative vehicle for integrating geologic analogs into multiple reservoir models. In: Grammer GM et al. (editors). AAPG memoir: integration of outcrop and modern analog data in reservoir models; 2002.

[6] CIS, Groundwater in drinking water protected areas - the implementation challenge of the water framework directive. common implementation strategy for the water framework directive. Tech. rep., Guidance Document No. 16, produced by Working Group C; 2007.

[7] Cole B, Silliman S. Capture zones for passive wells in heterogeneous unconfined aquifers. Ground Water 1997;35(1):92-8.

[8] Cooper $\mathrm{H}$, Jacob C. A generalized graphical method for evaluating formation constants and summarizing well-field history. Trans Am Geophys Union 1946;27(4):526-34.

[9] Darcel C, Bour O, Davy P, de Dreuzy JR. Connectivity properties of twodimensional fracture networks with stochastic fractal correlation. Water Resour Res 2003;39(10):1272.

[10] de Marsily G. Flow and transport in fractured rocks: connectivity and scale effect, Invited paper. In: International symposium on the hydrogeology of rocks of low permeability, Tucson AZ (USA), January, 1985.

[11] Fernandez-Garcia D, Sanchez-Vila X, Illangasekare TH. Convergent-flow tracer tests in heterogeneous media: combined experimental-numerical analysis for determination of equivalent transport parameters. J Contam Hydrol 2002;57(1-2):129-45.

[12] Fernandez-Garcia D, Illangasekare TH, Rajaram H. Differences in the scale dependence of dispersivity and retardation factors estimated from forcedgradient and uniform flow tracer tests in three- dimensional physically and chemically heterogeneous porous mediaart. no. w03012. Water Resour Res 2005;41(3):3012.

[13] Feyen L, Caers J. Quantifying geological uncertainty for flow and transport modeling in multi-modal heterogeneous formations. Adv Water Resour 2006;29(6):912-29.

[14] Fogg G. Groundwater flow and sand body interconnectedness in a thick, multiple-aquifer system. Water Resour Res 1986;22(5):679-94.

[15] Gelhar LW, Axness CL. 3-dimensional stochastic-analysis of macrodispersion in aquifers. Water Resour Res 1983;19(1):161-80.

[16] Gomez-Hernandez J, Wen X. To be or not to be multi-Gaussian? a reflection on stochastic hydrogeology. Adv Water Resour 1998;21(1):47-61.

[17] Guadagnini A, Franzetti S. Time-related capture zones for contaminants in randomly heterogeneous formations. Ground Water 1999;37(2):253-60.

[18] Guimera J, Carrera J. A comparison of hydraulic and transport parameters measured in low-permeability fractured media. J Contam Hydrol 2000;41(34):261-81.

[19] Harbaugh A, Banta E, Hill M, McDonald M. Modflow-2000 the us geological survey modular ground-water model-user guide to modularization concepts and the ground-water flow process. US Geological Survey Open-File Report 0092; 2000. p. 121.

[20] Kerrou J, Renard P, Franssen HJH, Lunati I. Issues in characterizing heterogeneity and connectivity in non-multiGaussian media. Adv Water Resour 2008;31:147-59.

[21] Knudby C, Carrera J. On the relationship between indicators of geostatistical, flow and transport connectivity. Adv Water Resour 2005;28(4):405-21.

[22] Knudby C, Carrera J. On the use of apparent hydraulic diffusivity as an indicator of connectivity. J Hydrol 2006;329(3-4):377-89.

[23] LaBolle EM, Fogg GE. Role of molecular diffusion in contaminant migration and recovery in an alluvial aquifer system. Transport Porous Media 2001;42(12):155-79.

[24] Liu GS, Zheng CM, Gorelick SM. Evaluation of the applicability of the dualdomain mass transfer model in porous media containing connected highconductivity channels. Water Resour Res 2007;43.

[25] Manzocchi T. The connectivity of two-dimensional networks of spatially correlated fractures. Water Resour Res 2002;38(9):1162.

[26] Meier PM, Carrera J, Sanchez-Vila X. An evaluation of jacob's method for the interpretation of pumping tests in heterogeneous formations. Water Resour Res 1998;34(5):1011-25.

[27] Neuweiler I, Vogel HJ. Upscaling for unsaturated flow for non-gaussian heterogeneous porous media. Water Resour Res 2007;43(3).

[28] Odling NE. Scaling and connectivity of joint systems in sandstones from western norway. J Struct Geol 1997;19(10):1257-71.

[29] Poeter E, Townsend P. Assessment of critical flow path for improved remediation management. Ground Water 1994;32(3):439-47.

[30] Riva M, Guadagnini L, Guadagnini A, Ptak T, Martac E. Probabilistic study of well capture zones distribution at the lauswiesen field site. J Contam Hydrol 2006;88(1-2):92-118.

[31] Sanchez-Vila X, Carrera J. Directional effects on convergent flow tracer tests. Math Geol 1997;29(4):551-69.

[32] Sanchez-Vila X, Rubin Y. Travel time moments for sorbing solutes in heterogeneous domains under nonuniform flow conditions. Water Resour Res 2003;39(4).

[33] Sanchez-Vila X, Carrera J, Girardi J. Scale effects in transmissivity. J Hydrol 1996;183(1):1-22.

[34] Sanchez-Vila X, Meier PM, Carrera J. Pumping tests in heterogeneous aquifers: an analytical study of what can be obtained from their interpretation using jacob's method. Water Resour Res 1999;35(4):943-52.

[35] Sanchez-Vila X, Guadagnini A, Carrera J. Representative hydraulic conductivities in saturated groundwater flow. Rev Geophys 2006;44(3): RG3002. 
[36] Schad H, Teutsch G. Effects of the investigation scale on pumping test-results in heterogeneous porous aquifers. J Hydrol 1994;159(1-4):61-77.

[37] Shafer JM. Reverse pathline calculation of time-related capture zones in nonuniform flow. Ground Water 1987;25(3):283-9.

[38] Strebelle S. Sequential simulation drawing structures from training images. PhD dissertation, Stanford, Stanford University; 2000. 187 p.

[39] Strebelle S. Conditional simulation of complex geological structures using multiple-point statistics. Math Geol 2002;34(1):1-21.

[40] Trinchero P, Sanchez-Vila X, Copty N, Findikakis A. A new method for the interpretation of pumping tests in leaky aquifers. Ground Water 2008;46(1):133-43.

[41] Varljen MD, Shafer JM. Assessment of uncertainty in time-related capture zones using conditional simulation of hydraulic conductivity. Ground Water $1991 ; 29(5): 737-48$.

[42] Varni M, Carrera J. Simulation of groundwater age distributions. Water Resour Res 1998;34(12):3271-81
[43] Vogel HJ. Topological characterization of porous media. Morphol Condens Matter: Phys Geometry Spatially Complex Syst 2002;600:75-92.

[44] Western AW, Bloschl G, Grayson RB. Toward capturing hydrologically significant connectivity in spatial patterns. Water Resour Res 2001;37(1): 83-97.

[45] Willmann M, Carrera J, Sanchez-Vila X. Transport upscaling: effects of heterogeneity on memory functions. Water Resour Res, submitted for publication.

[46] Zheng CM, Gorelick SM. Analysis of solute transport in flow fields influenced by preferential flowpaths at the decimeter scale. Ground Water 2003; 41(2):142-55.

[47] Zinn B, Harvey CF. When good statistical models of aquifer heterogeneity go bad: a comparison of flow, dispersion, and mass transfer in connected and multivariate gaussian hydraulic conductivity fields. Water Resour Res 2003;39(3):1051. 